\title{
Out of Hospital Pediatric Cardiac Arrest: Prospective Study from Riyadh, Saudi Arabia
}

\author{
Hashim M. Bin Salleeh ${ }^{1 *}$, Manal Al Tom ${ }^{1}$, Yasmeen Ahmed ${ }^{1}$, \\ William J. Leggio ${ }^{2}$ and Nabeel F. Abdulqader ${ }^{3}$ \\ ${ }^{1}$ Deparment of Emergency Medicine, College of Medicine, \\ King Saud University, Riyadh, Kingdom of Saudi Arabia. \\ ${ }^{2}$ EMS Education, School of Pharmacy and Allied Health, \\ Creighton University, Omaha, Nebraska, USA. \\ ${ }^{3}$ Prince Sultan Bin Abdulaziz, College for Emergency Medical Services, \\ King Saud University, Riyadh, Kingdom of Saudi Arabia \\ http://dx.doi.org/10.13005/bbra/2071
}

(Received: 27 December 2015; accepted: 03 February 2016)

\begin{abstract}
The objective of this study was to identify the incidence and clinical variables of out of hospital non-traumatic pediatric cardiac arrest in Riyadh, Kingdom of Saudi Arabia. A prospective protocol was developed to gather information relating to out of hospital non-traumatic pediatric cardiac arrests that were transported to King Khalid University Hospital in Riyadh, Saudi Arabia. A total of 23 patients were enrolled in the study and ranged from 10 days to 13 years old. Males represented $69.6 \%$ of the enrolled patients. The patient's home was reported as where $87 \%$ of the patients went into cardiac arrest. Only 1 patient was transported to the emergency department by ambulance with majority of others being transported by private vehicle. Asystole was the most common cardiac rhythm upon arrival. Eleven cases received cardiopulmonary resuscitation with 6 of these patients having a known history of chronic illness. Cardiopulmonary resuscitation was not performed on 12 of the patients and all of these but two had a known chronic illness. None of the patients enrolled in this study had a spontaneous return of circulation. Data from this study support other studies on the low survival rate for out of hospital pediatric cardiac arrest. Recommendations for public education and further research at a national level on the topic were supported by the limitations and findings.
\end{abstract}

Key words: Out-of-hospital, cardiac arrest, pediatric, Saudi Arabia

Out of hospital care in the Kingdom of Saudi Arabia is still developing as ambulance services remain to be provided by both a large public organization, and public and private hospitals. Most arrest victims in Riyadh are transported to the hospital in private vehicles by individuals who are rarely trained in cardiopulmonary resuscitation (CPR). ${ }^{1}$ A literature review on the topic of out of hospital nontraumatic pediatric arrests in Riyadh, Saudi Arabia identified one study on out of hospital cardiac arrests at a single tertiary care hospital in Riyadh,

\footnotetext{
* To whom all correspondence should be addressed.
}

Saudi Arabia from 1989 through $1995 .{ }^{1}$ A total of 95 patients, 61 adults and 34 children, were included in the study. ${ }^{1}$ Twenty-seven out of the 34 pediatric patients arrested outside of the hospital and none receive out of hospital $\mathrm{CPR}^{1}$. Of these 27 children: 8 arrested due to respiratory infection, 5 from congenital heart disease, 3 drowned and 2 arrested due to seizure activity. ${ }^{1}$ Only 2 of these pediatric patients survived to hospital discharge, neither were transported by ambulance and both suffered severe neurological deficits. ${ }^{1}$

Pediatric out of hospital cardiac arrest has been well associated with poor outcomes in the absence of bystander CPR. Though uncommon, pediatric out of hospital cardiac arrests have a poor rate of survival and negative neurological 
outcomes for those that do survive., ${ }^{2,3}$ Common causes of out of hospital cardiac arrest in pediatric patients were reported as sudden infant death syndrome, trauma and respiratory arrest. ${ }^{2,4}$ Cardiac causes have been reported as the more common cause for out of hospital pediatric cardiac arrests, though it is uncommon to have an identifiable cause of cardiac arrest during the initial phase of resuscitation. ${ }^{1,5-6}$ The incidence of non-traumatic out of hospital pediatric cardiac arrests have been reported to be 8-9 cases per 100,000 compared to 9-19 traumatic pediatric arrests per $100,000 .^{3-4,6-7}$ Up to $88 \%$ of pediatric cardiac arrests are reported to occur in non-public locations such as a private residence. ${ }^{3}$ Witnessed pediatric cardiac arrest with onsite CPR has been associated with better survival rates. ${ }^{8}$ In addition, providing out of hospital CPR, based on the 2010 American Heart Association standards, along with integrated care has been reported to result in several folds improvement in resuscitation outcomes. ${ }^{7}$

\section{METHODS}

This study received approval from the Institutional Review Board at King Saud University College of Medicine (12/3292/IRB). This study aimed to identify the incidence and clinical variables of out of hospital non-traumatic pediatric cardiac arrests presenting at King Khalid University Hospital (KKUH) in Riyadh, Saudi Arabia. A prospective cohort design was applied to study non-traumatic pediatric out of hospital cardiac arrests that presented at the Emergency Department (ED) of KKUH between January 2012 and September 2013. KKUH is located on the King Saud University campus in the capital city of Riyadh, Saudi Arabia. KKUH is an urban based tertiary care university hospital located near major roads, businesses and residential neighborhoods. KKUH pediatric ED annually treats approximately 51,000 patients of different acuity levels.

All non-traumatic out of hospital cardiac arrests brought to pediatric ED at KKUH was included in this study. ED physicians and nurses whom were all certified in American Heart Association Pediatric Advance Life Support cared for cardiac arrest patients completed a data collection form designed for this study. An investigator of this study reviewed each data collection form. Adult patients, cardiac arrests in the ED, patients suffering from traumatic cardiac arrest, and patients with do not resuscitate orders were excluded from this study.

SPSS (IBM, Armonk, NY, United States) with statistical package for social sciences version 20 was used to report the mean, standard deviation (SD), percentages, tables, and figures to express the data.

\section{RESULTS}

A total of 23 patients met the requirements to be enrolled in this study. These patients were captured without seasonal variation. The ages of the enrolled patients ranged from 10 days to 13 years of age with a mean age of 4 years (SD \pm 4 years). Males represented $69.6 \%$ of the patients. Other patient characteristics are reported in Table 1. The commonest recorded rhythm in the ED during cardiac arrest was asystole.

Table 2 reports $87 \%$ of the cardiac arrests occurred at the patient's home. Family was the most prevalent mode of arrival to the ED at $87 \%$ and only 1 patient arrived by EMS. Of the 23 patients only one case reported out of hospital CPR being performed prior to ED arrival.

CPR was performed on 11 cases in the ED as reported in Table 3. None of the patients had a return of spontaneous circulation. Eight patients received 3 cycles of epinephrine with a single case receiving 4 cycles and a second case receiving 5 cycles. Of the 11 cases in which CPR was performed, 6 had a chronic illness such as cerebral palsy with complications, congenital heart disease,

Table 1. Patient Characteristics

\begin{tabular}{lc}
\hline Variable & Result \% (n) \\
\hline Gender & \\
Male & $69.6(16)$ \\
Female & $30.4(7)$ \\
Chronic Illness & \\
Yes & $69.6(16)$ \\
No & $30.4(7)$ \\
On Medications & \\
Yes & $47.8(11)$ \\
No & $52.2(12)$ \\
\hline
\end{tabular}


Table 2. Summary of Pre-Arrival Characteristics

\begin{tabular}{lc}
\hline Variable & Result \% (n) \\
\hline Arrest Site & $87.0(20)$ \\
Home & $8.7(1)$ \\
Public Place & $4.3(1)$ \\
Rehabilitation Center & \\
Brought by & $87.0(20)$ \\
Family & $4.3(1)$ \\
EMS & $4.3(1)$ \\
Rehabilitation center & $4.3(1)$ \\
Transferred from another & \\
health care facility & $4.3(1)$ \\
Out of Hospital CPR Provided & $95.7(22)$ \\
Yes & \\
No & \\
\hline CPR - cardiopulmonary resuscitation EMS - emergency \\
medical services
\end{tabular}

Table 3. Summary of ED Care

\begin{tabular}{lc}
\hline Variable & Result \% (n) \\
\hline CPR Provided & \\
Yes & $43.5(10)$ \\
No & $56.5(13)$ \\
First recorded rhythm & \\
Asystole & $91.3(21)$ \\
Ventricular Tachycardia & $4.3(1)$ \\
Other & $4.3(1)$ \\
ED Disposition & $100(23)$ \\
Death Announced & $0(0)$ \\
Return of spontaneous Circulation & \\
CPR - cardiopulmonary resuscitation & \\
\hline
\end{tabular}

and arrhythmias. No chronic illnesses were reported for the other 5 patients. CPR was not performed on the other 12 enrolled cases. Ten of these 12 patients had chronic diseases already discussed. The other 2 patients were not reported to have chronic illness.

A total of 7 patients did not have a chronic illness reported and therefore were considered to be healthy pediatric patients. Figure 1 illustrates 5 of the 7 patients without chronic illness received CPR. The two patients with chronic illness that did not receive CPR were 6-month-old males. These two study participants were brought to the ED atleast one hour after being observed to be unresponsive by their parents. Both patients had a rectal temperature of $38.5 \mathrm{C}$ and the cause of death

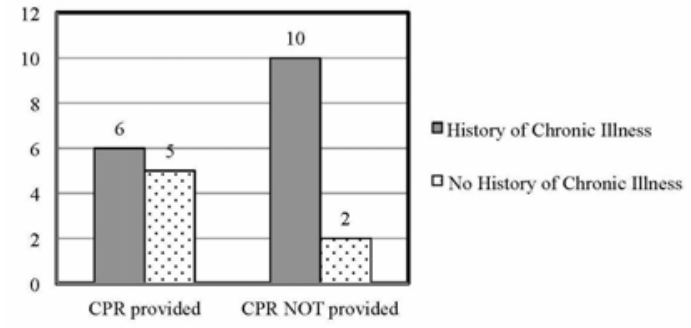

Fig. 1. Comparison of CPR provided to patients with and without history of Chronic illness

was thought to be septic shock. Clinical data indicated cardiac arrest to be caused by septic shock in 5 cases and near drowning, aspiration and cardiac origins for the other remaining cases.

\section{DISCUSSION}

The majority of patients enrolled in this study did not arrive to the pediatric ED by EMS, arrested at home and did not receive bystander CPR. These findings supported previous findings on most arrest victims in Riyadh, Saudi Arabia being transported to hospitals by private vehicles, ${ }^{1}$ and supported reports on the majority of pediatric cardiac arrests occurring at home ${ }^{3}$. None of the patients in this study had a spontaneous return of circulation thus supporting literature reporting poor survival rates in out of hospital pediatric cardiac arrests. ${ }^{1-3}$

This study reported the rate of CPR bring provided to pediatric patients in cardiac arrest with a known history of chronic illness compared to those without. The comparison discovered patients with a history of chronic illness, such as cerebral palsy with complications, congenital heart disease and arrhythmias, were less likely to receive CPR than healthy children. However, almost $40 \%$ of the patients with known chronic illness enrolled in this study did still receive CPR. Within the clinical data is a notable frequency of suspected septic shock as the cause of cardiac arrest.

This study was limited to a single university hospital in Riyadh, Saudi Arabia. Limited data was collected about prehospital arrival variables such as time from arrest to hospital arrival, why EMS system was not utilized and response time if activated. An additional limitation is a definitive cause of arrest was not confirmed by 
autopsy because of culture standards that limit performing an autopsy to suspected legal cases.

Further studies are necessary at the national level to identify variables related to pediatric out of hospital cardiac arrest. Support for public education programs is important to improve onsite CPR and use of EMS system for out of hospital pediatric cardiac arrests. Additionally, hospitals need to develop guidelines on when to perform pediatric CPR that considers culture, clinical presentation, and history of known chronic illness.

\section{CONCLUSION}

This study is relevant for emergency medical and out of hospital professionals in Riyadh, Saudi Arabia. Though limited to a single hospital in Riyadh, the data collected in this study yield a low survival rate for non-traumatic out of hospital pediatric cardiac arrest patients in Riyadh, Saudi Arabia, which was consistent with low survival rates for out of hospital pediatric cardiac arrests previously discovered. Findings of this study allowed for recommendations for research and support for public education related outing of hospital CPR to be made. This study renews the recommendations made in 1999 for community education in CPR, the need for EMS capable of providing prehospital stabilization, and means to provide early defibrillation in out of hospital environments in Saudi Arabia. ${ }^{1}$

\section{REFERENCES}

1. Conroy, K.M., Jolin ,S.W. Cardiac arrest in Saudi Arabia: A 7-year experience in Riyadh. J Emerg Med. 1999; 17(4):617-23.

2. Kudenchuk, P.J., Redshaw, J.D., Stubbs, B.A., Fahrenbruch, C.E., Dumas, F., Phelps, R., Blackwood, J., Rea, T.D., Eisenberg, M.S. Impact of changes in resuscitation practice on survival and neurological outcome after out-ofhospital cardiac arrest resulting from nonshockable arrhythmias. Circulation. 2012; 125(14):1787-94.

3. Tagami, T., Hirata, K., Takeshige, T., Matsui, J., Takinami, M., Satake, M., Satake, S., Yui, T., Itabashi, K., Sakata, T., Tosa, R., Kushimoto, S., Yokota, H., Hirama, H. Implementation of the fifth link of the chain of survival concept for out-of-hospital cardiac arrest. Circulation. 2012; 126(5):589-97.

4. Hinchey, P.R., Myers, J.B., Lewis, R., De, Maio, V.J., Reyer, E., Licatese, D., Zalkin, J., Snyder, G. Improved out-of-hospital cardiac arrest survival after the sequential implementation of 2005 AHA guidelines for compressions, ventilations, and induced hypothermia: the Wake County experience. Ann Emerg Med. 2010; 56(4):348-57.

5. Berdowski, J., Berb, R.A., Tijssen, J.G.P., Koster, R.W. Global incidences of out-ofhospital cardiac arrest and survival rates: Systematic review of 67 perspective studies. Resuscitation. 2010; 81(11):1479-87.

6. Stiell, I.G., Wells, G.A., Field, B.J., Spaite, D.W., De Maio, V.J., Ward, R., Munkley, D.P., Lyver, M.B., Luinstra, L.G., Campeau, T., Maloney, J., Dagnone, E. Improved out-of-hospital cardiac arrest survival through the inexpensive optimization of an existing defibrillation program: OPALS study phase II. Ontario Prehospital Advanced Life Support. JAMA. 1999; 281(13):1175-81.

7. Iwami, T., Nichol, G., Hiraide ,A., Hayashi, Y., Nishiuchi, T., Kajino, K., Morita, H., Yukioka, H., Ikeuchi ,H., Sugimoto, H., Nonogi, H., Kawamura, T. Continuous Improvements in "Chain of Survival" Increased Survival After Out-of-Hospital Cardiac Arrests. A Large-Scale Population-Based Study. Circulation. 2009;119:728-34.

8. Wissenberg, M., Lippert, F.K., Folke ,F., Weeke, P., Hansen, C.M., Christensen, E.F., Jans, H., Hansen, P.A., Lang-Jensen, T., Olesen, J.B., Lindhardsen, J., Fosbol, E.L., Nielsen, S.L., Gislason, G.H., Kober, L., Torp-Pedersen, C. Association of national initiatives to improve cardiac arrest management with rates of bystander intervention and patient survival after out-of-hospital cardiac arrest. JAMA. 2013; 310(13):1377-84. 